\title{
Relationship of cystatin C, Hs-CRP, neutrophil-lymphocyte ratio and platelet-lymphocyte ratio with isolated oligohydramnios
}

\author{
Ozlem Erten ${ }^{1}(\mathbb{D}$, Aysun Tekeli Taskomur² \\ ${ }^{1}$ Kutahya Health Sciences University, Kutahya, Turkey \\ ${ }^{2}$ Amasya University, School of Medicine, Amasya, Turkey
}

\begin{abstract}
Objectives: We evaluated inflammatory parameters in pregnant women with isolated oligohydramnios.

Material and methods: This prospective cross-sectional study enrolled 54 pregnant with isolated oligohydramnios (IO) and 54 matched by gestational week, healthy pregnant with normal amniotic fluid. Maternal plasma levels of cystatin C, hs-CRP, neutrophil-lymphocyte ratios (NLR), platelet-lymphocyte ratios (PLR), and pregnancy outcomes were compared between two groups.

Results: Cystatin C, hs-CRP, and PLR were significantly higher in the IO group than that in the control group $(\mathrm{p}<0.05)$. In the IO group, the rate of primary cesarean section, fetal distress, neonates with meconium-stained, and need for neonatal intensive care unit was higher, and Apgar scores were significantly lower than those in the control group ( $p<0.05)$. There was no significant difference between the groups for meconium-stained neonate rates and the intensive care unit's need in the late-term (410/7-416/7 weeks). Cystatin C, hs-CRP, and PLR were significantly higher in the IO group than the control group $(p<0.05)$. Cystatin $C$ was positively correlated with the need for neonatal intensive care and negatively correlated with Apgar scores. The PLR was positively correlated with the rate of meconium-stained neonates $(p<0.05)$. Cystatin C and hs-CRP had significant value in predicting IO $(p<0.05)$.

Conclusions: Maternal serum levels of Cystatin $C$ and hs-CRP may support the diagnosis and prediction of perinatal outcomes as possible biochemical markers in 10 cases. In particular, a high level of cystatin C may indicate the need for neonatal intensive care and low Apgar scores. In addition, late-term 10 may show similar results in meconium and neonatal intensive care needs compared to without oligohydramnios.
\end{abstract}

Key words: cystatin C; Hs-CRP; neutrophil-lymphocyte ratio; oligohydramnios; platelet-lymphocyte ratio

Ginekologia Polska 2022; 93, 11: 881-888

\section{INTRODUCTION}

Oligohydramnios is a common clinical complication in pregnancy. Its incidence ranges from $1 / 60$ to $1 / 750$. Hill et al. [1] reported an average of $0.9 \%$. There are many factors that play a role in its etiology. Some of these factors are fetal genitourinary system anomalies, placental insufficiency, maternal hypertensive, renal diseases, rupture of amniotic membranes, and history of maternal drug use such as angiotensin-converting enzyme inhibitor and nonsteroidal anti-inflammatory drugs [2]. Isolated oligohydramnios (IO) also called idiopathic oligohydramnios accounts for about half of the cases of oligohydramnios [2]. It is thought that this situation is caused by decreased placental perfusion and hypoxia, which leads to decreased of fetal urine production [3].

The diagnose of oligohydramnios is made, when the amniotic fluid index is $<5 \mathrm{~cm}$ or single pocket measurement is $<2 \mathrm{~cm}$ measured by two-dimensional ultrasound [4]. The incidence of oligohydramnios increases in the term periods of pregnancy. Its incidence rises to $15-20 \%$ at 42 weeks of gestation [5]. Amniotic fluid surrounds the fetus after the first few weeks of pregnancy. It has important functions in the growth and development of the fetus [6]. Fetal, maternal, and obstetric adverse effects may occur in oligohydramnios. Adverse outcomes such as admission to the neonatal intensive care unit (NICU), cesarean delivery

\section{Corresponding author:}

Ozlem Erten

Kutahya Health Sciences University, Department of Obstetrics and Gynecology, Turkey

e-mail: ozlem.erten@ksbu.edu.tr 
for fetal distress, and meconium aspiration syndrome have been shown to increase even in low-risk pregnancies with oligohydramnios [7].

Studies have shown a close relationship between oligohydramnios and intrauterine growth retardation, small gestational age, prolonged labor, low Apgar score, and an increase in neonatal intensive care $[8,9]$. Therefore, evaluation of amniotic fluid has been used as an integral component of obstetric screening and evaluation of fetal well-being for many years. However, it is not possible to measure the amniotic fluid in terms of volume and shape because it is constantly dynamic due to maternal and fetal factors [10]. Amniotic fluid measurement methods applied in the clinic are subjective and determining the amniotic fluid volume is directly related to the doctor's experience [11].

Cystatin $C$ is an extracellular cysteine protease inhibitor present in many tissues and fluids in the body. Cysteine is involved in many processes such as inflammation and tumor metastasis by regulating protease activity [12]. CRP is a very sensitive acute phase reactant. CRP blood levels can be measured precisely (up to values below $0.2 \mathrm{mg} / \mathrm{L}$ ) with new methods, i.e., hs-CRP. Recent studies have shown that increased CRP levels have a predictive value in diseases involving chronic inflammation such as cardiovascular diseases [13]. Studies of the neutrophil-lymphocyte ratio (NLR) and platelet-lymphocyte ratio (PLR) have shown a significant association with cancer and many diseases with inflammation; these parameters are beginning to be considered as possible inflammation markers $[14,15]$.

The pathophysiology of oligohydramnios is not fully known, but it is thought to develop secondary to chronic stress [16]. Oligohydramnios develops without fetal renal agenesis or obstructive uropathy and is thought to be due to fetal response to chronic stress $[16,17]$. It is caused by a decrease in fetal perfusion and urinary output secondary to the fact that the blood flow due to chronic stress is directed to vital organs such as the brain, heart, adrenal gland, and kidneys [18]. Fetal hypoxia [19] and acidosis [9] develop due to impaired fetal perfusion. Therefore, an increase in fetal and neonatal morbidity and mortality is observed in oligohydramnios [17]. There are studies arguing that poor perinatal outcomes which have been shown to be associated with oligohydramnios do not increase in isolated oligohydramnios. Other studies argue the opposite [20]. These poor perinatal outcomes are thought to be due to the relationship between oligohydramnios and placental insufficiency [21]. As a result, fetal perfusion disorder and secondary fetal hypoxia and acidosis may develop from placental insufficiency [22]. A cellular inflammatory response occurs from fetal hypoxia [23]. Both placental and fetal inflammation develop if due to acidosis [24]. Inflammation that occurs in the fetus and placenta may cause an inflammatory response in the mother [25].

\section{Objectives}

In this study, we wanted to evaluate the relationship of oligohydramnios with levels of cystatin C, hs-CRP NLR, and PLR. They are associated with inflammatory diseases in recent studies $[26,27]$. We suspect that they play a role in the pathophysiology of oligohydramnios. Thus, we wanted to evaluate whether these parameters could be used as clinical markers due to their association with oligohydramnios, whether they could support the diagnosis of oligohydramnios as inflammatory parameters, and whether these parameters have value in terms of obstetric outcomes.

\section{MATERIAL AND METHODS}

Ethical approval for this study was obtained from the Ethics Committee of Amasya University (protocol \#46). Those included in the study were selected from pregnant women over 32 weeks of gestation who applied to the Obstetrics and Gynecology outpatient clinics of Amasya Sabuncuoğlu Şerefeddin Training and Research Hospital between 8 November 2019 and 15 November 2020. The diagnosis of oligohydramnios was made in two ways: amniotic fluid index (AFI) less than $5 \mathrm{~cm}$ or less than $2 \mathrm{~cm}$ in the deepest single pocket (SDP) [10]. Patients diagnosed with oligohydramnios with both methods were included in the study. Patients diagnosed with oligohydramnios according to one method and not diagnosed with oligohydramnios according to the other method were not included in the study. In terms of dynamic changes in amniotic fluid, amniotic fluid was measured at two different times during the day (2-4 hours after the first measurement). A Mindray DC-7 ultrasound device was used to measure the amniotic fluid. The amniotic fluid evaluation was performed by a single clinician (A.T.T.) to minimize differences between measurements.

Pregnant women with chronic disease (such as diabetes mellitus, Addison's disease, adrenocortical insufficiency) or obstetric disease (such as gestational diabetes, hypertensive diseases of pregnancy, intrauterine growth retardation, premature rupture of membranes) that may trigger inflammation (such as collagen tissue disease, maternal infectious diseases) were excluded. Pregnant women with a history of drug use (such as a nonsteroidal anti-inflammatory or angiotensin-converting enzyme inhibitor) and with fetal congenital anomalies were not included in the study.

The study group consisted of 54 pregnant women with IO who meet the study criteria and 54 healthy pregnant women with normal amniotic fluid at the same gestational week as the matched control group. Of the pregnant women who were diagnosed with IO, 16 were 41 weeks, 25 were 40 weeks, eight were 39 weeks, two were 38 weeks, one was 36 weeks, and two were 34 weeks. All pregnant women were included in the study after being informed about the study and obtaining their consent. In terms of demo- 
graphic characteristics of both groups, age, parity, height, weight, educational status, previous cesarean section, and previous non-cesarean abdominal surgery were questioned.

The obstetric outcomes of both groups (delivery type, primary cesarean rate, cesarean section indications, low birth weight, neonates with meconium, neonatal intensive care need, as well as $1^{\text {st }}$ and $5^{\text {th }}$ minute Apgar scores) were compared. The relationship of cystatin C, hs-CRP, NLR, and PLR with obstetric results was investigated. Here, 41 -week pregnant women in the groups were evaluated separately in terms of neonatal intensive care need and rates of babies with meconium.

Blood samples were collected into one hemogram tube and two biochemistry tubes to evaluate cystatin C, hs-CRP, neutrophils, lymphocytes, thrombocytes, as well as neutrophil-lymphocyte and thrombocyte-lymphocyte ratios. Neutrophil, lymphocyte and thrombocyte levels were measured with laser optics (X N-1000, Siemens, Japan), Hs-CRP with an immunoturbidimetric test (Cobas C 702, Roche, Japan), and cystatin $C$ level with an immunonephelometric test (Dade Behring, Germany).

\section{Power analysis}

The sample size was determined with the $G$ * Power 3.1 program via a statistician who made a statistical analysis of the study similar to Figueroa et al. [28] using a disease prevalence of $0.7 \%$. We analyzed the number of women diagnosed with new pregnancy in one year at the health institution where the study was conducted ( $n=1600-1700)$ as well as effect width $(\mathrm{d}=0.71)$ and a two-tailed hypothesis method 36. The confidence interval was determined as $95 \%$, and the margin of error was $5 \%$. As a result of the calculation, we determined that there should be 53 women in the control group and 53 in the study group or 106 women in total.

\section{Statistical analysis}

The data were analyzed using the SPSS 21.0 program. Whether the continuous variables were suitable for normal distribution according to the groups was evaluated with the Kolmogorov-Smirnov test. A Mann-Whitney U test was used to compare variables that did not conform to a normal distribution. One-way analysis of variance (ANOVA) was used to compare normally distributed data according to three or more groups, and the Kruskal-Wallis test was used to compare non-normally distributed data. The Chi-square test or Fisher's exact probability test was used to determine whether the frequency distributions of categorical variables were homogeneously distributed among groups. A Chi-square test was used to test the relationships between categorical variables, and the $Z$ test (Bonferroni method) was used in the post hoc analysis of variables that were statistically different between groups. The $p<0.05$ was considered statistically significant. Spearman's rho correlation coefficient was used to examine the relationship between non-normally distributed quantitative variables. ROC curve analysis was used to find threshold values. The sensitivity, specificity, positive predictive, and negative predictive values of these limits were calculated in the presence of significant limit values. Cases with Type 1 error level below 5\% were considered statistically significant upon evaluation of the area under the curve.

\section{RESULTS}

The groups were similar in terms of demographic characteristics. Comparison of the groups according to demographic parameters is shown in Table 1. Cystatin C, hs-CRP, and PLR levels were significantly higher in pregnant women with oligohydramnios than the control group ( $p<0.05$ ). No statistically significant difference was observed between the groups in terms of NLR. The distribution of Cystatin C, hs-CRP, PLR, and NLR values among groups is shown in Table 2.

\begin{tabular}{|c|c|c|c|c|}
\hline & & 10 group $(n=54)(\%)$ & Control group $(n=54)(\%)$ & $\mathbf{p}$ \\
\hline Age [year] & & $28.19 \pm 5.36$ & $27.87 \pm 6.07$ & 0.776 \\
\hline BMI $\left[\mathrm{kg} / \mathrm{m}^{2}\right]$ & & $29.85 \pm 3.91$ & $30.56 \pm 3.76$ & 0.336 \\
\hline \multirow{2}{*}{ Parity } & Nulliparous & 21 (38.9\%) & 18 (33.3\%) & 0.689 \\
\hline & Multiparous & $33(61.1 \%)$ & $36(66.7 \%)$ & \\
\hline \multirow{4}{*}{ Education } & Primary school & $10(18.5 \%)$ & $12(22.2 \%)$ & 0.767 \\
\hline & Middle School & $9(16.7 \%)$ & $6(11.1 \%)$ & \\
\hline & High school & $22(40.7 \%)$ & $25(46.3 \%)$ & \\
\hline & University & $13(24.1 \%)$ & $11(20.4 \%)$ & \\
\hline PCS & & $7(13.0 \%)$ & $9(16.7 \%)$ & 0.588 \\
\hline PAS & & $8(14.8 \%)$ & 11 (20.4\%) & 0.614 \\
\hline
\end{tabular}

$\mathrm{p}$ - values were calculated with the Independent test (age, BMI) and Chi-square test; $1 \mathrm{O}$ — isolated oligohydramnios; PAS — previous abdominal surgery (except cesarean); PCS - previous cesarean surgery 
Table 2. Comparison of laboratory results of the groups

\begin{tabular}{|l|l|l|l|}
\hline & 10 group $(\mathbf{n}=\mathbf{5 4})$ & Control group $(\mathbf{n = 5 4})$ & $\mathbf{p}$ \\
\hline Cystatin C [mg/L] & $0.69 \pm 0.06$ & $0.65 \pm 0.06$ & $\mathbf{0 . 0 0 6}$ \\
\hline Hs-CRP $[\mathrm{mg} / \mathrm{L}]$ & $2.75 \pm 0.62$ & $2.47 \pm 0.77$ & $\mathbf{0 . 0 4 1}$ \\
\hline Neutrophils $\left[\times 10^{9} / \mathrm{L}\right]$ & $4.52 \pm 0.81$ & $4.24 \pm 0.80$ & 0.078 \\
\hline Lymphocytes $\left[\times 10^{9} / \mathrm{L}\right]$ & $2.29 \pm 0.55$ & $2.43 \pm 0.64$ & 0.235 \\
\hline Platelets $\left[\times 10^{9} / \mathrm{L}\right]$ & $305.50 \pm 59.66$ & $288.37 \pm 68.10$ & 0.167 \\
\hline NLR & $2.08 \pm 0.61$ & $1.89 \pm 0.68$ & 0.134 \\
\hline PLR & $140.82 \pm 43.70$ & $124.56 \pm 37.02$ & $\mathbf{0 . 0 3 9}$
\end{tabular}

$\mathrm{p}$ - values were calculated with the Independent test; $1 \mathrm{O}$ — isolated oligohydramnios; NLR — neutrophil to lymphocyte ratio; PLR — platelet to lymphocyte ratio

Table 3. Sensitivity, specificity, positive predictive, and negative predictive values for cystatin C, Hs-CRP, NLR, and PLR

\begin{tabular}{|l|l|l|l|l|l|}
\hline & AUC $(\mathbf{9 5} \mathrm{Cl})$ & $\mathbf{p}$ & Cut-off & Sensitivity & Specificity \\
\hline Cystatin C [mg/L] & $0.636(0.531-0.74)$ & $\mathbf{0 . 0 1 5}$ & $\geq 0.675$ & 0.574 & 0.574 \\
\hline Hs-CRP $[\mathrm{mg} / \mathrm{L}]$ & $0.656(0.549-0.762)$ & $\mathbf{0 . 0 0 5}$ & $\geq 2.615$ & 0.704 & 0.667 \\
\hline NLR & $0.589(0.482-0.697)$ & 0.109 & - & - & - \\
\hline PLR & $0.607(0.501-0.714)$ & 0.054 & - & - & -
\end{tabular}

$\mathrm{p}$ - values were calculated with the ROC analyses; AUC — area under the curve; NLR — neutrophil to lymphocyte ratio; PLR — platelet lymphocyte ratio

Cystatin C, hs-CRP, NLR, and PLR were evaluated with Receiver Operator Characteristics Curve (ROC). The area under the curve in ROC was statistically significant for cystatin C and hs-CRP ( $p<0.05$ ). However, there was no statistical significance of the area under the curve in the ROC for NLR and PLR ( $p>0.05$ ) (Tab. 3).

When both groups were compared in terms of obstetric results, primary cesarean section, fetal distress, neonates with meconium, and neonatal intensive care need were higher in the oligohydramnios group, but these were not statistically significant $(p>0.05)\left(\right.$ Tab. 4). The $1^{\text {st }}$ and $5^{\text {th }}$ minute Apgar scores were significantly lower in the IO group compared to the control group $(p<0.05)$ (Tab. 4). There was no difference between the groups in terms of birth weight.

The relationship of cystatin C, hs-CRP, NLR, and PLR parameters with obstetric results were also evaluated. PLR was positively correlated with rates of meconium neonates $(r=0.274$, $p=0.004)$. Cystatin C was positively correlated with neonatal intensive care need and negatively correlated with 1 st and 5th minute Apgar scores $(r=0.237, p=0.013)$. Correlation analyses according to the groups are shown in Table 5.

Since meconium is more expected in the late-term period, meconium-stained infants' rates and the NICU needs were also compared between the groups in the late-term. Evaluation of 41-week pregnant women in terms of meconium rates and neonatal intensive care needs showed no significant difference between the groups (Tab. 6).

Gestational week distribution according to NICU needs: seven infants at 41 weeks and 8 at 40 weeks were in the
IO group (n: 15). Three infants at 41 weeks, 3 at 40 weeks, and one at 39 weeks were in the control group (n: 7).

Gestational week distribution of meconium-stained infants: six infants at 41 weeks, four at 40 weeks, one at 39 weeks, and one infant at 38 weeks were in the IO group (n: 12). Three infants were at 41 weeks and three at 40 weeks in the control group (n: 6).

\section{DISCUSSION}

We evaluated maternal serum levels of the inflammation parameters cystatin C, hs-CRP, NLR, and PLR to determine whether the fetal and placental inflammation that develops in isolated oligohydramnios causes an inflammatory response in the mother. Cystatin C, hs-CRP, and PLR levels were significantly higher in the oligohydramnios group than the control group. Significantly higher values of the aforementioned parameters suggest that there is an inflammatory process in the pathophysiology of oligohydramnios and that a maternal inflammatory response may occur against this inflammation. There was a significant area under the curve (AUC) in the ROC of cystatin C and hs-CRP.This suggests that these two parameters may be suitable for use as diagnostic decision-makers in oligohydramnios $(p<0.05)($ Tab. 3). In addition, when the obstetric results of both groups were compared, the $1^{\text {st }}$ and $5^{\text {th }}$ minute Apgar scores were significantly lower in the IO group versus the control group $(p<0.05)$. Cystatin C elevation was positively correlated with neonatal intensive care need and negatively correlated with $1^{\text {st }}$ and $5^{\text {th }}$ minute Apgar scores. Although PLR did not have 
Table 4. Comparison of obstetric outcomes of groups

\begin{tabular}{|c|c|c|c|c|}
\hline & & 10 group $(n=54)(\%)$ & Control group $(n=54)(\%)$ & p \\
\hline Birth weight [g] & & $3141.11 \pm 478.20$ & $3288.43 \pm 484.2$ & 0.115 \\
\hline \multirow{2}{*}{ Type of delivery } & Vaginal & $34(72.2 \%)$ & $39(72.2 \%)$ & 0.304 \\
\hline & Cesarean & $20(37.0 \%)$ & $15(27.8 \%)$ & \\
\hline \multirow{6}{*}{ Birth week [week] } & $34-34^{6}$ week & $2(3.7 \%)$ & $2(3.7 \%)$ & 1.00 \\
\hline & $36-36^{6}$ week & $1(1.9 \%)$ & $1(1.9 \%)$ & \\
\hline & 38-386 week & $2(3.7 \%)$ & $2(3.7 \%)$ & \\
\hline & $39-39^{6}$ week & $8(14.8 \%)$ & $8(14.8 \%)$ & \\
\hline & $40-40^{6}$ week & $25(46.3 \%)$ & $25(46.3 \%)$ & \\
\hline & $41-41^{6}$ week & $16(29.6 \%)$ & $16(29.6 \%)$ & \\
\hline Primary cesarean & & $13(42.1 \%)$ & $6(11.1 \%)$ & 0.072 \\
\hline \multirow{4}{*}{ Cesarean indication } & Fetal distress & $10(50.0 \%)$ & $3(20.0 \%)$ & 0.092 \\
\hline & Previous cesarean & $7(35.0 \%)$ & $9(60.0 \%)$ & \\
\hline & CPD & $1(5.0 \%)$ & $3(20.0 \%)$ & \\
\hline & Prolonged labor & $2(10.0 \%)$ & $0(0.0 \%)$ & \\
\hline Low birth weight & & $4(7.4 \%)$ & $3(5.6 \%)$ & 0.696 \\
\hline Meconium & & $12(22.2 \%)$ & $6(11.1 \%)$ & 0.121 \\
\hline Need of NICU & & $15(27.8 \%)$ & $7(13.0 \%)$ & 0.056 \\
\hline $1^{\text {st }}$ minute AS & & $7.89 \pm 1.14$ & $8.43 \pm 0.83$ & 0.006 \\
\hline $5^{\text {st }}$ minute AS & & $9.09 \pm 0.78$ & $9.54 \pm 0.60$ & 0.001 \\
\hline
\end{tabular}

AS - apgar score; CPD — cephalopelvic dystocia; IO — isolated oligohydramnios; NICU — Neonatal Intensive Care Unit; $\mathrm{p}$ - values were calculated with the independent test (APGAR scores, birth weight) and Chi-square test

\begin{tabular}{|c|c|c|c|c|c|}
\hline & & Cystatin C [mg/L] & Hs-CRP [mg/L] & NLR & PLR \\
\hline \multirow{2}{*}{ Low birth weight } & $\mathrm{R}$ & 0.034 & -0.042 & 0.038 & 0.054 \\
\hline & $\mathrm{P}$ & 0.723 & 0.670 & 0.693 & 0.578 \\
\hline \multirow{2}{*}{ Primary cesarean } & $\mathrm{R}$ & 0.101 & 0.156 & 0.006 & 0.061 \\
\hline & $P$ & 0.298 & 0.106 & 0.953 & 0.533 \\
\hline \multirow{2}{*}{ Fetal distress } & $\mathrm{R}$ & 0.177 & 0.129 & -0.026 & 0.033 \\
\hline & $\mathrm{P}$ & 0.067 & 0.184 & 0.788 & 0.732 \\
\hline \multirow{2}{*}{ Meconium-stained } & $\mathrm{R}$ & 0.128 & -0.095 & 0.112 & 0.274 \\
\hline & $P$ & 0.186 & 0.326 & 0.247 & 0.004 \\
\hline \multirow{2}{*}{$1^{\text {st }}$ minute Apgar Score } & $\mathrm{R}$ & 0.237 & -0.054 & 0.105 & 0.097 \\
\hline & $\mathrm{P}$ & 0.013 & 0.580 & 0.279 & 0.319 \\
\hline \multirow{2}{*}{$5^{\text {th }}$ minute Apgar Score } & $\mathrm{R}$ & -0.288 & -0.015 & -0.106 & -0.121 \\
\hline & $P$ & 0.003 & 0.877 & 0.274 & 0.213 \\
\hline \multirow{2}{*}{ Neonatal intensive care need } & $\mathrm{R}$ & 0.237 & -0.054 & 0.105 & 0.097 \\
\hline & $P$ & 0.013 & 0.580 & 0.279 & 0.319 \\
\hline
\end{tabular}

NLR - neutrophil to lymphocyte ratio; P — values were calculated with the Spearman's rho correlation test; PLR - platelet lymphocyte ratio; R - Spearman's rrho correlation coefficient

decision-maker validity, it had a positive correlation with the meconium rate.

While ultrasonography is sufficient to evaluate the normal volumes of amniotic fluid, it is poor at identify- ing abnormal volumes [18]. In addition, the experience of the doctor, the fetal position, the possibility of temporary changes in amniotic fluid, and the existence of different ultrasound criteria that diagnose anomalous amniotic fluid 
Table 6. Comparison of both groups at the $41^{0 / 6}-41^{6 / 7 s t}$ gestational week in terms of neonatal intensive care need and meconium-stained rates

\begin{tabular}{|c|c|c|c|c|c|}
\hline \multicolumn{6}{|c|}{ Comparison of 41-week gestation of both groups in terms of neonatal intensive care needs } \\
\hline & 10 group NICU (-) n (9) & 10 group NICU (+) n (7) & $\begin{array}{l}\text { Control group NICU (-) } \\
\text { n (13) }\end{array}$ & $\begin{array}{l}\text { Control group NICU (+) } \\
\text { n (3) }\end{array}$ & p \\
\hline Cystatin C [mg/L] & $0.69 \pm 0.07$ & $0.73 \pm 0.06$ & $0.66 \pm 0.09$ & $0.67 \pm 0.01$ & 0.344 \\
\hline $\mathrm{Hs}-\mathrm{CRP}[\mathrm{mg} / \mathrm{L}]$ & $2.57 \pm 0.56$ & $2.42 \pm 0.81$ & $2.63 \pm 0.90$ & $2.67 \pm 0.10$ & 0.955 \\
\hline NLR & $1.72 \pm 0.43$ & $1.76 \pm 0.35$ & $2.09 \pm 0.79$ & $2.23 \pm 0.05$ & 0.354 \\
\hline PLR & $138.04 \pm 43.59$ & $139.41 \pm 48.71$ & $139.36 \pm 46.13$ & $140.82 \pm 13.39$ & 0.985 \\
\hline \multicolumn{6}{|c|}{ Comparison of 41-week gestation of both groups in rates of meconium } \\
\hline & $\begin{array}{l}10 \text { group Meconium (-) } \\
\mathrm{n}(10)\end{array}$ & $\begin{array}{l}10 \text { group Meconium (+) } \\
\mathrm{n}(6)\end{array}$ & $\begin{array}{l}\text { Control group Meconium (-) } \\
\text { n (13) }\end{array}$ & $\begin{array}{l}\text { Control group } \\
\text { Meconium (+) n (3) }\end{array}$ & p \\
\hline Cystatin C [mg/L] & $0.68 \pm 0.07$ & $0.74 \pm 0.07$ & $0.66 \pm 0.09$ & $0.67 \pm 0.01$ & 0.321 \\
\hline $\mathrm{Hs}-\mathrm{CRP}[\mathrm{mg} / \mathrm{L}]$ & $2.64 \pm 0.63$ & $2.28 \pm 0.70$ & $2.63 \pm 0.90$ & $2.67 \pm 0.10$ & 0.891 \\
\hline NLR & $1.64 \pm 0.40$ & $1.89 \pm 0.31$ & $2.09 \pm 0.79$ & $2.23 \pm 0.05$ & 0.264 \\
\hline PLR & $116.03 \pm 33.08$ & $176.32 \pm 34.55$ & $139.36 \pm 46.13$ & $140.82 \pm 13.39$ & 0.052 \\
\hline
\end{tabular}

$1 \mathrm{O}$ - solated oligohydramnios; NICU — neonatal intensive care unit; NLR - neutrophil-to-lymphocyte ratio; $\mathrm{p}$ - values were calculated with the Kruskal-Wallis test and one-way analysis of variance test; PLR - platelet lymphocyte ratio

volume led to a decrease in the reliability of ultrasound [29]. For this reason, we used ultrasound evaluation from the same physician to provide both AFI and SDP results and made measurements twice a day.

Accuracy rate can reach up to $87 \%$ when measured with the ultrasound index made by measuring three dimensions [30]. It has been suggested that invasive methods such as indicator dilution techniques give the most accurate measurement, but they are not practical for clinical use [19]. Therefore, existing methods continue to be used, and we used both (AFI, SDP).

The main clinical importance of oligohydramnios is due to the increase in fetal and neonatal morbidity and mortality [17]. These poor outcomes are thought to be due to placental insufficiency developing in oligohydramnios [21]. In addition, decreased uteroplacental flow may cause an increase in the release of chemokines and activation of neutrophils in the later stages of pregnancy. This is more common in healthy pregnancies and can overstimulate an inflammatory response [31]. Platelets, like neutrophils, increase the secretion of cytokines at the onset of inflammation, and increased cytokines contribute to increased inflammation by enhancing new neutrophil and platelet synthesis [32]. In light of these studies, we think that it is clinically important to investigate the relationship between the inflammatory process in the mother with $\mathrm{IO}$, inflammatory parameters, and obstetric outcomes. In the IO group, we found high levels of cystatin C, hs-CRP, and PLR among these inflammation markers support that an inflammatory process plays a role in the pathogenesis of oligohydramnios. Although the current literature is constantly developing, there is still limited information in terms of the pathophysiology and management of $I O$.
While some studies suggest increased risks in $\mathrm{IO}$, other studies report the opposite [2]. In our study we tried to exclude negative obstetric outcomes due to secondary factors (such as diabetes, hypertension etc.). In addition, there is no method recommended to accurately predict perinatal morbidity and mortality in $\mathrm{IO}$, and there is no consensus for cut-off values [33]. Here, when the obstetric results of the groups were compared, the $1^{\text {st }}$ and $5^{\text {th }}$ minute Apgar scores were significantly lower in the IO group than the control group. When the correlation of these inflammatory markers with obstetric outcomes was evaluated, the PLR level was positively correlated with the number of neonates with meconium, and the level of cystatin C was positively correlated with the need for neonatal intensive care. Cystatin $C$ levels were negatively correlated with $1^{\text {st }}$ and $5^{\text {th }}$ minute Apgar scores. These results may support the idea that pregnant women with oligohydramnios with high cystatin C need to be more careful in terms of fetal well-being. This could contribute positively to obstetric outcomes by allowing the necessary preparations to be made in terms of intensive care conditions and Apgar scores. Oligohydramnios, meconium association, and the need for neonatal intensive care were associated more frequently in pregnant women at 41 weeks of gestation and were accepted as late-term in our study [34]. Thus, 41-week pregnant women were re-evaluated to reduce bias in terms of perinatal outcomes. We think that pregnant women in our study had $\mathrm{IO}$ cases even if they were late-term ( $41^{0 / 7}-41^{6 / 7}$ week); thus, they had similar intensive care needs and meconium rates as the control group. There was no difference in birth weight between the groups like the results of studies suggesting that isolated oligohydramnios is not associated with impaired fetal growth or increased risk of adverse perinatal outcomes [20]. 
The strength of our study is that this is the first study evaluating the relationship between inflammatory parameters and oligohydramnios. Cases were matched with controls, and mean birth weight was evaluated in $1 \mathrm{O}$ cases that were not complicated by intrauterine growth retardation; secondary factors were excluded in the comparison of obstetric results. The evaluation was also done for late-term weeks. Markers such as PLR and hs-CRP were used, which can be easily examined in the clinic. We suspect that it may be possible to use these to predict poor perinatal outcomes.

One limitation of our study was that while we did have sufficient statistical power, we could not perform a large series. In addition, a maternal comparison of inflammatory parameters could be done as well as a fetal comparison; however, we did not evaluate fetal levels in our study. This was similar to another study investigating the relationship of cystatin C in cases with intrauterine growth retardation [35].

\section{CONCLUSIONS}

In summary, cystatin C, hs-CRP, and PLR, increase in inflammation and were found to be higher in $I O$ especially when cystatin $\mathrm{C}$ and hs-CRP were statistically and significantly higher in ROC analysis. This suggests that they could be used as markers in both the diagnosis of oligohydramnios and in predicting perinatal outcomes in suspected cases. In addition, a high cystatin C level may indicate a poor prognosis in terms of fetal well-being. It may be clinically helpful to determine prognostic parameters that would support the diagnosis of $\mathrm{IO}$ and enable to take precautions in terms of the risks.

\section{Conflict of interest}

All authors declare no conflict of interest.

\section{REFERENCES}

1. Hill LM, Breckle R, Thomas ML, et al. Polyhydramnios: ultrasonically detected prevalence and neonatal outcome. Obstet Gynecol. 1987; 69(1): 21-25, indexed in Pubmed: 3540761.

2. Dorot $A$, Wainstock $T$, Sheiner $E$, et al. Isolated oligohydramnios and long-term neurological morbidity of the offspring. J Dev Orig Health Dis. 2020; 11(6): 648-652, doi: 10.1017/S2040174419000795, indexed in Pubmed: 31755400.

3. Underwood MA, Gilbert WM, Sherman MP. Amniotic fluid: not just fetal urine anymore. J Perinatol. 2005; 25(5): 341-348, doi: 10.1038/sj.jp.7211290, indexed in Pubmed: 15861199.

4. Magann EF, Sandlin AT, Ounpraseuth ST. Amniotic fluid and the clinical relevance of the sonographically estimated amniotic fluid volume: oligohydramnios. J Ultrasound Med. 2011; 30(11): 1573-1585, doi: 10.7863/jum.2011.30.11.1573, indexed in Pubmed: 22039031.

5. Phelan JP, Platt LD, Yeh SY, et al. The role of ultrasound assessment of amniotic fluid volume in the management of the postdate pregnancy. Am J Obstet Gynecol. 1985; 151(3): 304-308, doi: 10.1016/00029378(85)90291-1, indexed in Pubmed: 3881964.

6. Brace RA, Gilbert WM, Brace RA, et al. Amniotic fluid volume regulation: basal volumes and responses to fluid infusion or withdrawal in sheep. Am J Physiol. 1987; 252(2 Pt 2): R380-R387, doi: 10.1152/ajpregu.1987.252.2.R380, indexed in Pubmed: 3101523.

7. Rabie N, Magann E, Steelman S, et al. Oligohydramnios in complicated and uncomplicated pregnancy: a systematic review and meta-analysis. Ultrasound Obstet Gynecol. 2017; 49(4): 442-449, doi: 10.1002/uog.15929, indexed in Pubmed: 27062200.

8. Voxman EG, Tran S, Wing DA. Low amniotic fluid index as a predictor of adverse perinatal outcome. J Perinatol. 2002; 22(4): 282-285, doi: 10.1038/sj.jp.7210697, indexed in Pubmed: 12032790.

9. Feldman I, Friger $M$, Wiznitzer $A$, et al. Is oligohydramnios more common during the summer season? Arch Gynecol Obstet. 2009; 280(1): 3-6, doi: 10.1007/s00404-008-0848-4, indexed in Pubmed: 19031078.

10. Lim Kl, Butt K, Naud K, et al. Amniotic Fluid:Technical Update on Physiology and Measurement. J Obstet Gynaecol Can. 2017; 39(1): 52-58, doi: 10.1016/j.jogc.2016.09.012, indexed in Pubmed: 28062025.

11. Williams K. Amniotic fluid assessment. Obstet Gynecol Surv. 1993; 48(12): 795-800, doi: 10.1097/00006254-199312000-00005, indexed in Pubmed: 8309662.

12. Henskens YM, Veerman EC, Nieuw Amerongen AV. Cystatins in health and disease. Biol Chem Hoppe Seyler. 1996; 377(2): 71-86, doi: 10.1515/bchm3.1996.377.2.71, indexed in Pubmed: 8868064.

13. Kablak-Ziembicka A, Przewlocki T, Sokołowski A, et al. Carotid intima-media thickness, hs-CRP and TNF- $a$ are independently associated with cardiovascular event risk in patients with atherosclerotic occlusive disease. Atherosclerosis. 2011; 214(1): 185-190, doi: 10.1016/j.atherosclerosis.2010.10.017, indexed in Pubmed: 21067752.

14. Feng JF, Huang $Y$, Chen QX. Preoperative platelet lymphocyte ratio (PLR) is superior to neutrophil lymphocyte ratio (NLR) as a predictive factor in patients with esophageal squamous cell carcinoma. World J Surg Oncol. 2014; 12: 58, doi: 10.1186/1477-7819-12-58, indexed in Pubmed: 24641770.

15. Boilard E, Nigrovic PA, Larabee $K$, et al. Platelets amplify inflammation in arthritis via collagen-dependent microparticle production. Science. 2010; 327(5965): 580-583, doi: 10.1126/science.1181928, indexed in Pubmed: 20110505

16. Moore TR. Clinical assessment of amniotic fluid. Clin Obstet Gynecol. 1997;40(2):303-313, doi: 10.1097/00003081-199706000-00007, indexed in Pubmed: 9199842.

17. Sherer DM. A review of amniotic fluid dynamics and the enigma of isolated oligohydramnios. Am J Perinatol. 2002; 19(5): 253-266, doi: 10.1055/s-2002-33084, indexed in Pubmed: 12152144.

18. Gramellini D, Fieni $S$, Verrotti $C$, et al. Ultrasound evaluation of amniotic fluid volume: methods and clinical accuracy. Acta Biomed. 2004; 75 Suppl 1: 40-44, indexed in Pubmed: 15301289.

19. Nabhan AF, Abdelmoula YA. Amniotic fluid index versus single deepest vertical pocket: a meta-analysis of randomized controlled trials. Int J Gynaecol Obstet. 2009; 104(3): 184-188, doi: 10.1016/j.ijgo.2008.10.018, indexed in Pubmed: 19046586.

20. Zhang J, Troendle J, Meikle S, et al. Isolated oligohydramnios is not associated with adverse perinatal outcomes. BJOG. 2004; 111(3): 220-225, doi: 10.1111/j.1471-0528.2004.00060.x, indexed in Pubmed: 14961882.

21. Miremberg $\mathrm{H}$, Grinstein $\mathrm{E}, \mathrm{Herman} \mathrm{HG}$, et al. The association between isolated oligohydramnios at term and placental pathology in correlation with pregnancy outcomes. Placenta. 2020; 90: 37-41, doi: 10.1016/j. placenta.2019.12.004, indexed in Pubmed: 32056549.

22. Pereira S, Chandraharan E. Recognition of chronic hypoxia and pre-existing foetal injury on the cardiotocograph (CTG): Urgent need to think beyond the guidelines. Porto Biomed J. 2017; 2(4): 124-129, doi: 10.1016/j.pbj.2017.01.004, indexed in Pubmed: 32258602.

23. Wood CE, Keller-Wood M. Current paradigms and new perspectives on fetal hypoxia: implications for fetal brain development in late gestation. Am J Physiol Regul Integr Comp Physiol. 2019; 317(1): R1-R13, doi: 10.1152/ajpregu.00008.2019, indexed in Pubmed: 31017808.

24. Xu A, Matushewski B, Cao M, et al. The ovine fetal and placental inflammatory response to umbilical cord occlusions with worsening acidosis. Reprod Sci. 2015; 22(11): 1409-1420, doi: 10.1177/1933719115580994, indexed in Pubmed: 25878209.

25. Oh JW, Park CW, Moon KC, et al. The relationship among the progression of inflammation in umbilical cord, fetal inflammatory response, early-onset neonatal sepsis, and chorioamnionitis. PLoS One. 2019; 14(11): e0225328, doi: 10.1371/journal.pone.0225328, indexed in Pubmed: 31743377

26. Deyà-Martínez À, Fortuny C, Soler-Palacín P, et al. Cystatin C: a marker for inflammation and renal function among HIV-infected children and adolescents. Pediatr Infect Dis J. 2016; 35(2): 196-200, doi: 10.1097/INF.0000000000000960, indexed in Pubmed: 26479972.

27. Qin B, Ma N, Tang Q, et al. Neutrophil to lymphocyte ratio (NLR) and platelet to lymphocyte ratio (PLR) were useful markers in assess- 
ment of inflammatory response and disease activity in SLE patients. Mod Rheumatol. 2016; 26(3): 372-376, doi: 10.3109/14397595.2015.1091136, indexed in Pubmed: 26403379.

28. Figueroa L, McClure EM, Swanson J, et al. Oligohydramnios: a prospective study of fetal, neonatal and maternal outcomes in low-middle income countries. Reprod Health. 2020; 17(1): 19, doi: 10.1186/s12978-0200854-y, indexed in Pubmed: 32000798.

29. FokWY, Chan LY, Lau TK. The influence of fetal position on amniotic fluid index and single deepest pocket. Ultrasound Obstet Gynecol. 2006; 28(2): 162-165, doi: 10.1002/uog.2802, indexed in Pubmed: 16708416.

30. Magann EF, Perry KG, Chauhan SP, et al. The accuracy of ultrasound evaluation of amniotic fluid volume in singleton pregnancies: the effect of operator experience and ultrasound interpretative technique. J Clin Ultrasound. 1997; 25(5): 249-253, doi: 10.1002/(sici)1097-0096(199706 )25:5<249::aid-jcu5>3.0.co;2-d, indexed in Pubmed: 9314106.

31. Mellembakken JR, Aukrust $\mathrm{P}$, Hestdal K, et al. Chemokines and leukocyte activation in the fetal circulation during preeclampsia. Hypertension.
2001; 38(3): 394-398, doi: 10.1161/01.hyp.38.3.394, indexed in Pubmed: 11566911.

32. Mantovani A, Cassatella MA, Costantini $C$, et al. Neutrophils in the activation and regulation of innate and adaptive immunity. Nat Rev Immunol. 2011; 11(8): 519-531, doi: 10.1038/nri3024, indexed in Pubmed: 21785456.

33. Magann EF, Isler CM, Chauhan SP, et al. Amniotic fluid volume estimation and the biophysical profile: a confusion of criteria. Obstet Gynecol. 2000; 96(4): 640-642, doi: 10.1016/s0029-7844(99)00634-1, indexed in Pubmed: 11004374.

34. Peipert JF, Donnenfeld AE. Oligohydramnios: a review. Obstet Gynecol Surv. 1991; 46(6): 325-339, doi: 10.1097/00006254-199106000-00002, indexed in Pubmed: 2067755.

35. Malamitsi-Puchner A, Briana DD, Kontara L, et al. Serum cystatin C in pregnancies with normal and restricted fetal growth. Reprod Sci. 2007; 14(1): 37-42, doi: 10.1177/1933719106298196, indexed in Pubmed: 17636214. 